\title{
Early use of everolimus improved renal function after adult deceased donor liver transplantation
}

\author{
Jaehun YANG, Sunghae PARK, Young Ju OH, Sang Oh YUN, Manuel LIM, Ji Eun KWON, \\ Eun Sung JEONG, Jinsoo RHU, Gyu-Seong CHOI, Jong Man KIM*, Jae-Won JOH
}

Department of Surgery, Samsung Medical Center, Sungkyunkwan University School of Medicine, Seoul, Korea

Introduction: Tacrolimus (TAC) is a main therapy for liver transplantation (LT) patients, but it has side effects such as chronic nephrotoxicity that progressively aggravate renal function. The purpose of this study was to retrospectively compare the renal function between a TAC group and a combination of everolimus and reduced TAC (EVR-TAC) group after deceased donor liver transplantation (DDLT).

Methods: The study comprised 131 patients who underwent DDLT between January 2013 and April 2018 at our institution. They received TAC or EVR-TAC after DDLT. EVR was introduced between one and six months after DDLT.

Results: Thirty-six of 131 patients (27.5\%) received EVR-TAC. The incidence of chronic kidney disease $(\mathrm{CKD})\left(\mathrm{eGFR}<60 \mathrm{~mL} / 1.73 \mathrm{~m}{ }^{2}\right)$ in the EVR-TAC group was higher than in the TAC group (25\% vs. $8.4 \% ; p=0.019)$. Increasing serum creatinine $(\mathrm{n}=23,63.9 \%)$ was the most common cause for adding EVR to treatment of the post-transplant patients. There were no statistical differences in acute rejection and CKD between the two groups. The TAC trough level was significantly lower in the EVR-TAC group than in the TAC group, and the renal function of the EVR-TAC group was worse than that of the TAC group until one year after DDLT. However, the renal function of the EVR-TAC group improved and became similar to that of TAC group at 3 years post-transplant.

Conclusions: The present study suggests that EVR should be introduced as soon as possible after DDLT to reduce exposure to high doses of TAC to improve the renal function. 